OPEN ACCESS

Edited by:

Salvatore Savasta,

University of Pavia, Italy

Reviewed by:

Laura Muiño Mosquera,

Ghent University Hospital, Belgium

Marco Castori,

Home for Relief of Suffering

(IRCCS), Italy

Chiara Villa,

University of Milano-Bicocca, Italy

*Correspondence:

Yang Liu

tjetyyly@163.com

†These authors have contributed equally to this work

Specialty section:

This article was submitted to Neonatology,

a section of the journal

Frontiers in Pediatrics

Received: 12 November 2021

Accepted: 17 January 2022

Published: 17 February 2022

Citation:

Yan X, Shu J, Nie Y, Zhang Y, Wang P, Zhou W, Cui X and Liu Y (2022) Case

Report: Identification and Functional

Analysis of a Homozygous

Synonymous Variant in the PLOD1

Gene in a Chinese Neonatal With the

Ehlers-Danlos Syndrome.

Front. Pediatr. 10:813758

doi: 10.3389/fped.2022.813758

\section{Case Report: Identification and Functional Analysis of a Homozygous Synonymous Variant in the PLOD1 Gene in a Chinese Neonatal With the Ehlers-Danlos Syndrome}

\author{
Xiaodan Yan ${ }^{1,2+}$, Jianbo Shu ${ }^{3,4 \dagger}$, Yanyan $\mathrm{Nie}^{1 \dagger}$, Ying Zhang ${ }^{1,2}$, Ping Wang ${ }^{3,4}$, \\ Weiwei Zhou ${ }^{1,2}$, Xiaoyu Cui ${ }^{1,2}$ and Yang Liu ${ }^{1 *}$
}

\begin{abstract}
Department of Neonatology, Tianjin Children's Hospital (Tianjin University Children's Hospital), Tianjin, China, ${ }^{2}$ Graduate College of Tianjin Medical University, Tianjin, China, ${ }^{3}$ Tianjin Key Laboratory of Birth Defects for Prevention and Treatment, Tianjin, China, ${ }^{4}$ Tianjin Pediatric Research Institute, Tianjin Children's Hospital (Tianjin University Children's Hospital), Tianjin, China
\end{abstract}

Background: Kyphoscoliotic Ehlers-Danlos syndrome (kEDS; OMIM225400) is a rare autosomal recessive genetic disease caused by variants in the PLOD1 gene. This research was conducted to verify the disease-causing gene in a Chinese neonatal family with the EDS.

Methods: We recruited a Han Chinese neonate with PLOD1-related kEDS without kyphoscoliosis. Detailed clinical examination and laboratory tests were performed and whole exome sequencing (WES) was used to detect the pathogenic genes of the proband. In vivo experiments (reverse-transcription PCR, quantitative real-time PCR) and in vitro experiments (minigene analysis) were used to verify the function of variants suspected of affecting the splicing process. The effect of the splice site variant on the PLOD1 transcript was analyzed using splice prediction programs NetGene2 and Alternative Splice Site Predictor (ASSP).

Results: A homozygous synonymous variant c.1095C > T (p.Gly365, rs1032781250) in the PLOD1 gene was found and verified in the family with kEDS. This splicing variant resulted in a premature termination codon of exon 10 and affected the expression of the four bases GCGC.

Conclusion: Our research showed that the homozygous synonymous variant in PLOD1 was the pathogenic cause in the proband. The combined application of WES and functional studies verified the effect of uncertain gene variants on splicing, upgrading pathogenicity evidence, and determining the cause of disease. This is helpful for the early diagnosis and treatment of kEDS.

Keywords: PLOD1, Ehlers-Danlos syndrome, newborn, synonymous variant, splicing 


\section{INTRODUCTION}

Kyphoscoliotic Ehlers-Danlos syndrome (kEDS; OMIM 225400) is a rare autosomal recessive genetic disorder with an incidence of 1:100,000 that occurs early in life and mainly affects the musculoskeletal system (1). In 1998, the Villefranche etiology classified EDS into six major subtypes based on the severity of clinical symptoms, genetic patterns, and potential biochemical and molecular defects (2). According to the last classification of EDS, it is classified into 13 subtypes (3). kEDS is divided into two subtypes based on molecular pathology. The PLOD1 variant causes kEDS-PLOD1 and the FKBP14 variant causes kEDS-FKBP14. Moreover, kEDS is characterized by severe intraocular hypotension at birth, progressive kyphosis, apparent skin hyper-elasticity, severe joint hyperactivity, and dislocation (4).

Most kEDS patients have a variant in the PLOD1 gene, which encodes the collagen-modifying enzyme-lysine and 2oxyglutarate-5-dioxygenase 1 (PLOD1 or lysine hydroxylase 1) (3). Lysine hydroxylase 1 (LH1) is a posttranslational modification enzyme that plays an important role in the biosynthesis of collagen, it will through hydroxylation of helical lysyl residues in-Xaa-Lys-Gly-collagen sequences to hydroxylysyl residues, which serve as sites of attachment for carbohydrate units, and in the process of the formation of intra- and intermolecular collagen cross-links (5). Lack of LH1 can lead to insufficient hydroxylation of collagen-dependent amino residues, thereby affecting cross-linking and making the affected tissue unstable (6). Therefore, the lack of this enzyme leads to the production of lysyl-pyridinoline (LP) and hydroxylysylpyridinoline (HP) in the body, which is then excreted abnormally via urine (7). The LH1 activity of skin fibroblasts in kEDS patients is $25 \%$ lower than that of normal people (8). To date, 56 variants have been reported in PLOD1 according to the EDS variant database (https://eds.gene.le.ac.uk/home.php?select_db= PLOD1).

In this research, whole exome sequencing (WES) was used to analyze the genetic cause in a family with kEDS$P L O D 1$. We performed additional functional analyses for variants with uncertain significance (VUS) suspected of affecting splicing through in vivo experiments (reverse transcription PCR, quantitative real-time PCR) and in vitro experiments (minigene analysis) and gathered further pathogenic evidence. Finally, the results of WES combined with functional analysis strongly support the diagnosis of kEDS-PLOD1 in the proband, who, to our knowledge, is the first neonatal Chinese kEDS-PLOD1 patient without kyphoscoliosis. We hope our results will provide a solid foundation for genetic counseling and disease diagnosis.

\section{MATERIALS AND METHODS}

\section{Subjects}

A Chinese family with kEDS-PLOD1, including three members, was recruited from Tianjin Children's Hospital in December 2020. This study has been approved by the ethics and human research committee of Tianjin Children's Hospital.

\section{DNA Extraction}

We obtained about $4 \mathrm{~mL}$ of EDTA anticoagulant peripheral blood samples from the patient and his parents after the parents signed the informed consent form. Genomic DNA was extracted using the Blood Genomic DNA Mini kit (cat. no. CW0541; CoWin Biosciences) according to the standard procedures. We used the NanoDrop ${ }^{\circledR} 2000$ spectrophotometer (Thermo Fisher Scientific, Inc.) to assess the absorbance ratio between 260 and $280 \mathrm{~nm}$ by extracting $1 \mu \mathrm{l}$ DNA. The measured concentrations of DNA were stored at $-20^{\circ} \mathrm{C}$.

\section{WES and Sanger Sequencing}

The proband was analyzed by WES and Sanger sequencing was conducted on the whole family by KingMED (Tianjin, China). We use Chromas software (version 1.62; Technelysium Pty. Ltd.) to contrast the sequencing data with the target sequences in NCBI (https://www.ncbi.nlm.nih.gov/) to confirm the variant.

\section{Evaluating the Significance of the PLOD1 Variants}

To evaluate the significance of the PLOD1 variants, we conducted reverse transcription $\mathrm{PCR}$ and quantitative real-time PCR (qPCR). Total RNA was extracted from lymphocytes and reverse transcribed with a reference operation by using the FastKing RT Kit (KR180123; Tiangen Biotech). The reverse transcription PCR reaction was followed by PCR amplification by using the forward primer ( $5^{\prime}$-CCTGGTCGGCGTGTTCATC- $\left.3^{\prime}\right)$ and reverse primer ( $3^{\prime}$-TGCGGTAGCTGTCTAGGGAGAG-5'). The thermocycling conditions were: initial denaturation at $95^{\circ} \mathrm{C}$ for $2 \mathrm{~min}$; followed by $35 \mathrm{cycles}$ of $95^{\circ} \mathrm{C}$ for $30 \mathrm{~s}, 60^{\circ} \mathrm{C}$ for $30 \mathrm{~s}$, and $72^{\circ} \mathrm{C}$ for $60 \mathrm{~s}$; and a final extension step at $72^{\circ} \mathrm{C}$ for $5 \mathrm{~min}$.

We then used BLAST (https://www.ncbi.nlm.nih.gov/tools/ primer-blast/index.cgi) to design primers for quantitative real-time PCR containing the variant site, forward primer ( $5^{\prime}$-GAGGTGCGGATGGCGAAT- $\left.3^{\prime}\right)$ and reverse primer $\left(3^{\prime}\right.$ GGGCAATGACGTTCTTGTTCT-5' $)$. A normal patient cDNA was also used as a control. The cDNA was diluted 10 times before being add to each reaction. The appropriate qPCR conditions were: $95^{\circ} \mathrm{C}$ for $5 \mathrm{~min}$ followed by 45 cycles of $95^{\circ} \mathrm{C}$ for $10 \mathrm{~s}$, $60^{\circ} \mathrm{C}$ for $10 \mathrm{~s}, 72^{\circ} \mathrm{C}$ for $10 \mathrm{~s}$, and $40^{\circ} \mathrm{C}$ for $30 \mathrm{~s}$ (fluorescent dye solubilization were $95^{\circ} \mathrm{C}$ for $5 \mathrm{~s}, 65^{\circ} \mathrm{C}$ for $60 \mathrm{~s}, 97^{\circ} \mathrm{C}$ for $1 \mathrm{~s}$ for 1 cycle). This reaction was conducted on an LC480-II thermal cycler (Roche Diagnostics) by performing the melting curve analysis and recording the cycle threshold $(\mathrm{Ct})$. We repeated the qPCR thrice and repeated each sample in quintuplicate to improve the error bars. All samples were run by GraphPad Prism software (version 8.0.1; GraphPad, USA). The mRNA expression level was calculated and normalized by using the $\Delta \mathrm{Ct}$ method relative to GAPDH.

\section{Minigene Constructions and Expression}

To confirm the effect of the c. $1095 \mathrm{C}>\mathrm{T}$ variant, in vitro analysis was performed by using a minigene splicing assay based on the pcDNA3.1 expression vector (Figures 1A,B). With the ClonExpress Ultra One Step Cloning Kit (Vazyme Biotech Co., Ltd.), the fragments with the wild-type (WT) alleles involving 9-11 exon, flanked by approximately 120-300 nucleotides of 


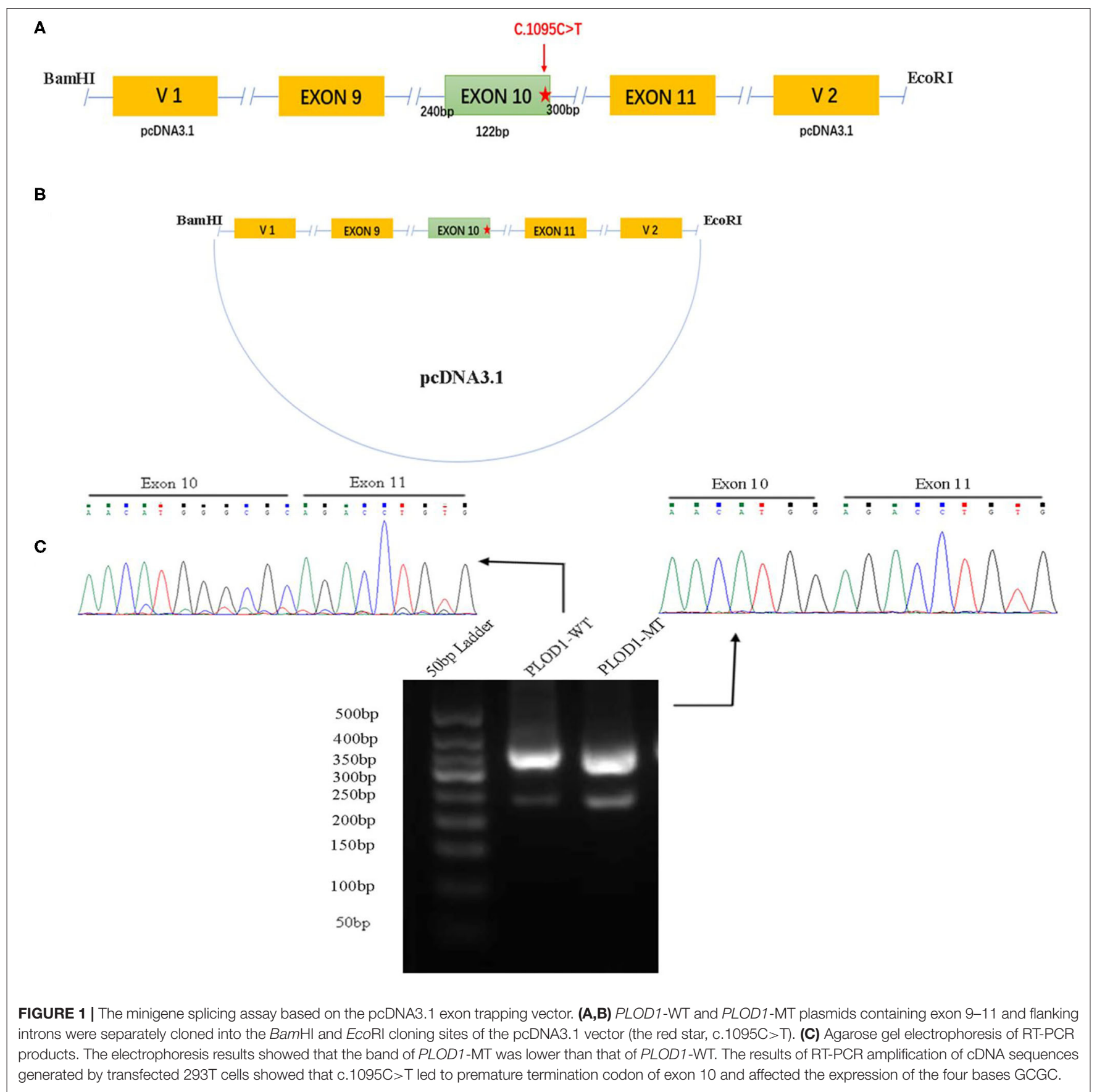

upstream intronic sequence and downstream intronic sequence, were cloned into the splicing vector pcDNA3.1 using specific primers linking the $B a m \mathrm{HI}$ and EcoRI restriction enzyme sites (BamHI: G^GATCC; EcoRI: G^AATTC). Primers were designed for each target fragment using CE Design software (version 1.04; Vazyme Biotech Co., Ltd.). The wild-type (WT) minigene plasmid was used as a template to generate the mutant-type (MT) minigene by using the Fast Site-directed Mutagenesis Kit (Tiangen Biotech [Beijing] Co., Ltd.). The wild and mutant type constructs were named PLOD1-WT and PLOD1-MT, respectively.
Both PLOD1-WT and PLOD1-MT were further transformed into Escherichia coli DH5 $\alpha$-competent cells (TaKaRa [Beijing] Co., Ltd.), confirmed by screening using conventional Sanger sequencing. Human epithelial kidney 293T (HEK 293T) cells were cultured in DMEM containing $10 \%$ fetal bovine serum (FBS), penicillin (100 U/L), and streptomycin (100 $\mathrm{mg} / \mathrm{L}$ ) at $37^{\circ} \mathrm{C}$ in a $5 \% \mathrm{CO}_{2}$ atmosphere. One day before transfection, both cells were transferred to a 6-well culture plate to grow to approximately $70-80 \%$ confluence in an antibiotic-free medium. HEK $293 \mathrm{~T}$ cells were then transfected with $2.5 \mu \mathrm{g}$ plasmid DNA (PLOD1-WT, PLOD1-MT, and 
empty PLOD1-control) using OPTI-MEM ${ }^{\circledR}$ IMedium and Lipofectamine 2000 (Invitrogen, Carlsbad, CA) according to the manufacturer's instructions. After $24 \mathrm{~h}$, the cells were harvested and total RNA was extracted with Trizol reagent (Tiangen Biotech). Total RNA was reverse-transcribed to cDNA using FastKing RT Kit (with gDNase) (Tiangen Biotech) by using the following vector-specific primers: forward primer (PLOD1-F, 5'-GATGAAGCTCTGCCCACGGT-3') and reverse primer (PLOD1-R, 3'-TTGTTCTGTTGGATCAGCAG-5'). Samples were heated to $95^{\circ} \mathrm{C}$ for $5 \mathrm{~min}$, followed by 34 cycles of DNA denaturation $\left(95^{\circ} \mathrm{C}\right.$ for $\left.60 \mathrm{~s}\right)$, annealing $\left(58^{\circ} \mathrm{C}\right.$ for $\left.30 \mathrm{~s}\right)$, and polymerization $\left(72^{\circ} \mathrm{C}\right.$ for $\left.30 \mathrm{~s}\right)$. After the last cycle, the samples were incubated for an additional $5 \mathrm{~min}$ at $72^{\circ} \mathrm{C}$. The PCR products were subsequently separated by electrophoresis on a $2.5 \%$ agarose gel, and the intensity of each band was quantified by ImageJ software. All transcripts were analyzed by sequencing.

\section{RESULTS}

\section{Subjects}

The patient was a 22-h-old male neonate and the second child of unrelated Chinese parents. He was born at $36^{+3}$ weeks of gestation through vaginal delivery, and the mother had a history of unknown spontaneous abortion at 19 weeks of pregnancy. The proband weighed $2,800 \mathrm{~g}$ at birth and measured $48 \mathrm{~cm}$ in length and had a head circumference of $34.5 \mathrm{~cm}$, with a normal Apgar score. Physical examination revealed no kyphoscoliosis, wrist sagging, excessive abduction of the bilateral hip joints, bipedal valgus and excessive flexion, muscle weakness, and swelling of hands and feet. The patient's primitive reflexes could not be elicited, and the mental response was weak. The patient's cranial magnetic resonance imaging (MRI) showed intraventricular hemorrhage and subarachnoid hemorrhage (Figures 2A,B). Facial examination showed bruises on the right eye socket, ears, and both sides of the nose, a $3 \times 3-\mathrm{cm}$ fluctuating mass on the top of the head, and a slightly higher palatal arch (Figures 2C,D). Ambulatory electroencephalography (AEEG) was abnormal, with increased trace discontinue (TD) and prolonged interburst interval (IBI). Echocardiography showed patent ductus arteriosus and patent foramen ovale. X-ray showed that sclerotin was normal and the patient did not have thoracic scoliosis on chest X-Ray. Electrophysiological examination revealed multiple peripheral nerve lesions, which improved after 13 days but still showed multiple damages. The serum creatine kinase (CK) level was $8505 \mathrm{U} / \mathrm{L}$ (normal, <310 U/L). CK-MB was $265 \mathrm{U} / \mathrm{L}$ (normal, <24 U/L). CK-MBmass was $202.9 \mathrm{ng} / \mathrm{mL}$ (normal, $<14.97 \mathrm{ng} / \mathrm{mL}$ ). These results suggested that the patient had myocardial damage. The Thyroid Stimulating Hormone (TSH) level was $7.377 \mathrm{mIU} / \mathrm{L}$ (normal, <5.33 mIU/L) and 25hydroxyvitamin D level was $5.9 \mathrm{ng} / \mathrm{mL}$ (normal, $>15 \mathrm{ng} / \mathrm{mL}$ ). Routine biochemical examination, acute phase reactants, blood and sputum culture results, neonatal blood and urine screening, blood gas analysis, and levels of lactic acid and serum immunoglobulin were normal.
A

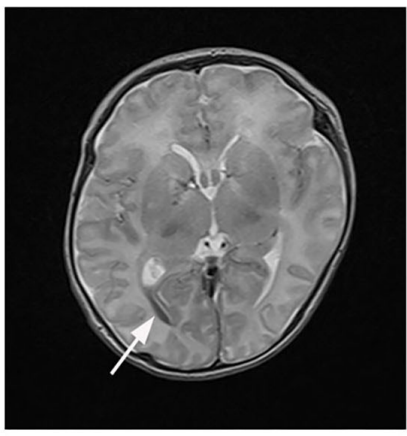

B

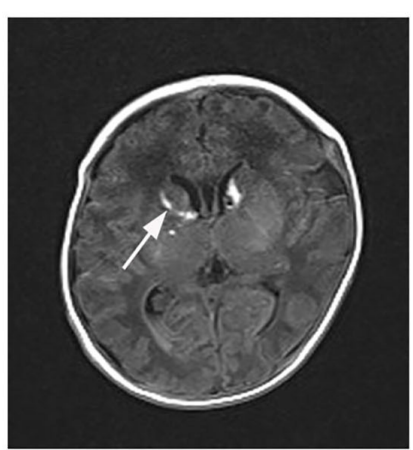

C

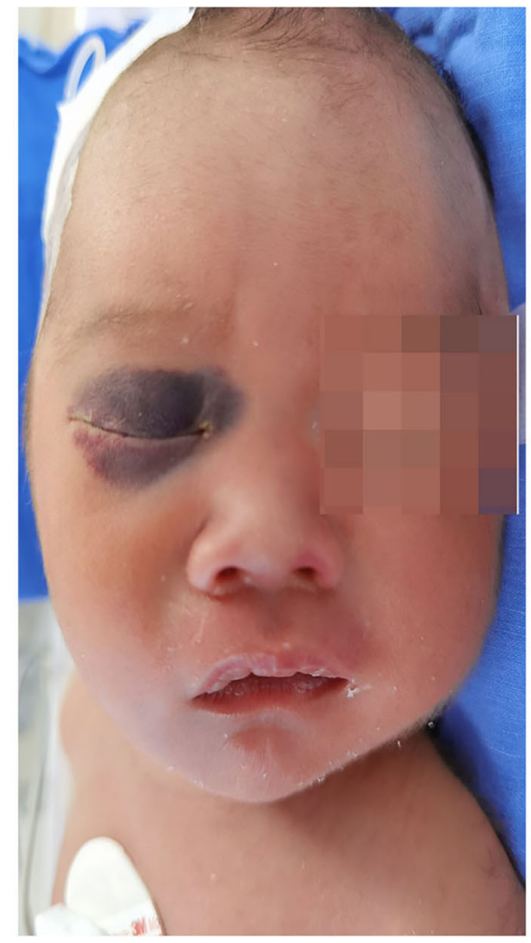

D

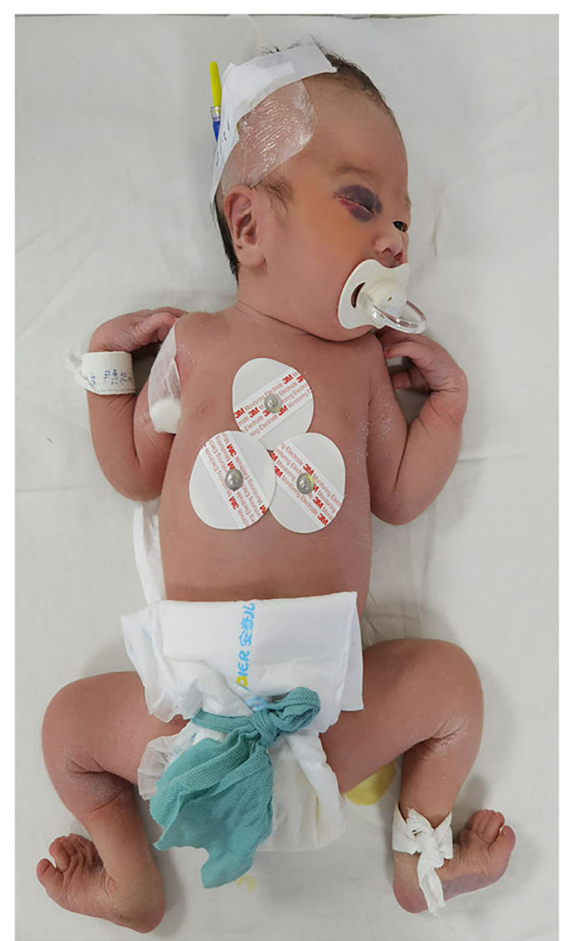

FIGURE 2 | Clinical features and imaging manifestations of proband. (A,B) Cranial MRI: intraventricular hemorrhage and subarachnoid hemorrhage (direction of arrow). (C) Facial bruises. (D) Wrist, excessive abduction of the bilateral hip joints, bipedal valgus, and excessive flexion. 


\section{Detection and Certification of the Variants}

The patient's WES showed that there was a homozygous synonymous variant on exon 10 of PLOD1 (NM_000302.3), c.1095C $>$ T (p.Gly365=). Based on the result of WES, we performed Sanger sequencing on the parents and the proband. The parents were all heterozygous carriers, which was in accordance with the autosomal recessive inheritance pattern (Figures 3A,B).

\section{Prediction of Pathogenicity}

We used NetGene2 Server (http://www.cbs.dtu.dk/services/ NetGene2/) to predict the c.1095C > T (p.Gly365=) variation that affects the splicing impact. The result was consistent with the pathogenicity site, which caused the premature termination codon of the exon, and the confidence score of the result was 0.95 (Figures 4A,B). We also used the Alternative Splice Site Predictor (ASSP) (http://wangcomputing.com/assp/index.html) to predict the variation, which conformed to the NetGene2 Server (Figures 4C,D).

\section{Variant Validation and Analysis}

RT-PCR verified that the variant affects the splicing, which caused the premature termination codon of exon 10 to affect the expression of the four bases of GCGC (Figure 5). These results are consistent with those of Sanger sequencing, NetGene2 Server, and ASSP.

The results of the qPCR revealed that the mRNA level of the proband was significantly lower than the normal control (NC) (Figure 6). The mRNA levels of parents were both half of the normal control, but both parents were phenotypically normal. These results collectively confirmed that the premature termination codon of exon 10 led to abnormal transcription of PLOD1 and caused disease.

\section{Minigene Analysis}

The PLOD1-WT and PLOD1-MT minigene constructs were validated in $293 \mathrm{~T}$ cells, where they produced the expected transcripts. The electrophoresis results showed that the amplified cDNA band corresponding to the spliced transcripts in the PLOD1-MT was lower than that in the PLOD1-WT (Figure 1C). In other words, the PLOD1-MT PCR produced a smaller cDNA product than the PLOD1-WT. This is likely because the expression of PLOD1-MT minigene led to a premature termination codon of exon 10 , which was verified by the sequence of cDNA-amplified products. It proved that the c.1095C $>\mathrm{T}$ variant was the cause of premature termination codon of exon 10.

\section{Genetic Variation Analysis of PLOD1 in the Patient}

The pathogenicity of PLOD1 variants in the proband was assessed based on the American College of Medical Genetics and Genomics (ACMG) standards and guidelines for the interpretation of sequence variants (9). The c.1095C $>\mathrm{T}$ variant is absent from controls in the Exome Sequencing Project, 1000 Genomes Project, or Exome Aggregation Consortium (PM2). In vitro or in vivo functional studies support a damaging effect on the gene (PS3). According to the splice prediction program, the $c .1095 \mathrm{C}>\mathrm{T}$ variant significantly affects splicing (PP3). The patient's phenotype or family history was highly specific for kEDS (PP4). As a result, the $\mathrm{c} .1095 \mathrm{C}>\mathrm{T}$ variant was classified as "likely pathogenic" based on the above evidence.

\section{DISCUSSION}

kEDS-PLOD1 is a rare autosomal recessive genetic disorder. According to the latest revision of the international standards of EDS, there are three main standards in the diagnosis of kEDSPLOD1 including congenital muscle weakness and congenital or early-onset of kyphosis (progressive or non-progressive);

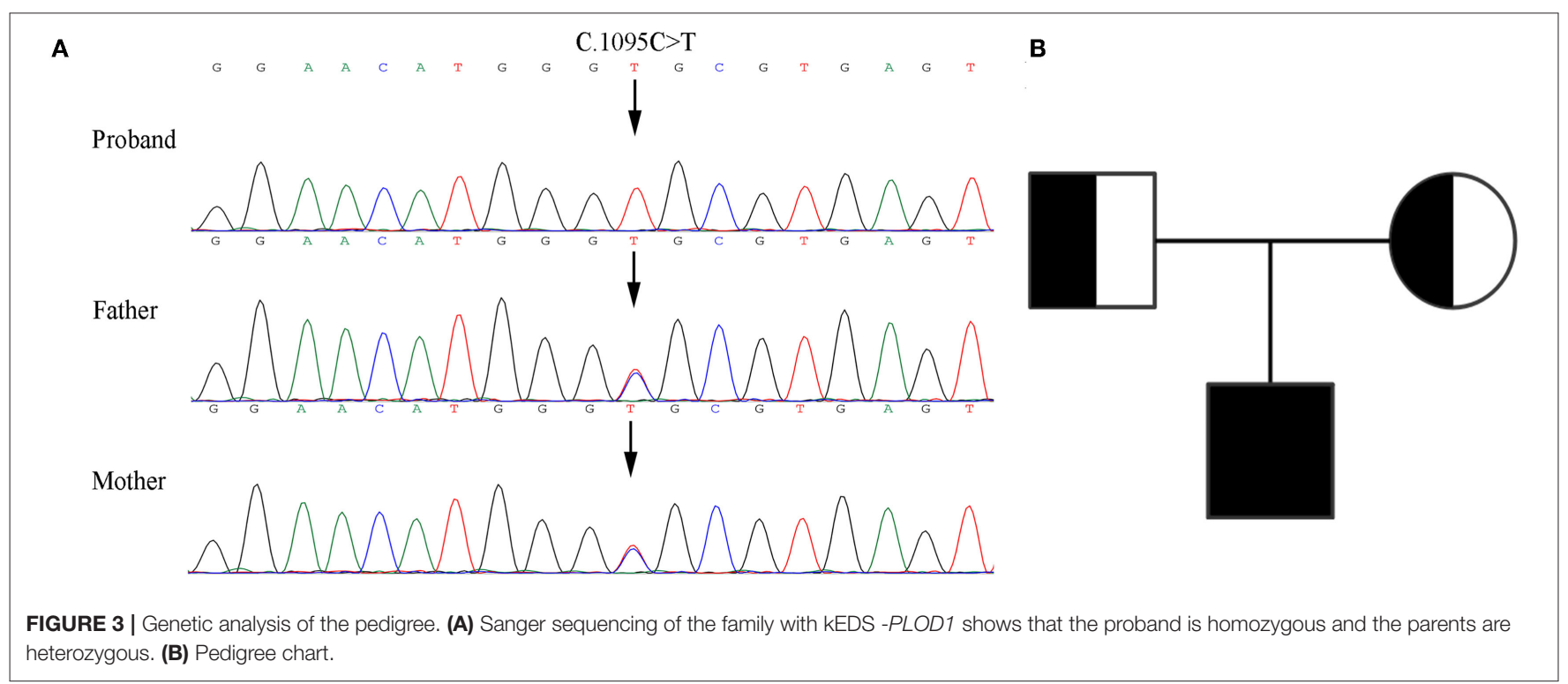


A Donor splice sites, direct strand

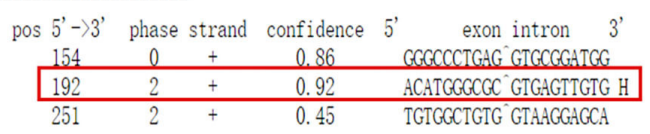

C

\begin{tabular}{|c|c|c|c|c|c|c|c|}
\hline \multirow[b]{2}{*}{ Position (bp) } & \multirow[b]{2}{*}{ Putative splice site } & \multirow[b]{2}{*}{ Sequence } & \multirow[b]{2}{*}{ Scoret } & & \multirow[b]{2}{*}{ Confidence** } \\
\hline & & & & Intron $G C^{*}$ & Alt/Cryptic & Constitutive & \\
\hline 90 & Alt. isoform/cryptic donor & CAAGGCTCAGgtggaagagt & 5.957 & 0.614 & 0.784 & 0.163 & 0.792 \\
\hline 122 & Alt. isoform/cryptic donor & ATGGCAGCGAgtaccagtct & 4.718 & 0.629 & 0.863 & 0.103 & 0.881 \\
\hline 141 & Alt. isoform/cryptic donor & TGTGAAGCTGgtgggecctg & 10.336 & 0.629 & 0.593 & 0.324 & 0.453 \\
\hline 153 & Alt. isoform/cryptic donor & GGGCCCTGAGgtgcggatgg & 11.186 & 0.600 & 0.528 & 0.381 & 0.279 \\
\hline 191 & Alt. isoform/cryptic donor & ACATGGGCGCgtgagttgtg & 8.321 & 0.614 & 0.658 & 0.272 & 0.587 \\
\hline 226 & Alt. isoform/cryptic acceptor & ccactgacagTGGGGGCAGG & 3.989 & 0.600 & 0.894 & 0.099 & 0.889 \\
\hline 250 & Constitutive donor & TGTGGCTGTGgtaaggagca & 10.383 & 0.543 & 0.317 & 0.583 & 0.456 \\
\hline
\end{tabular}

B

Donor splice sites, direct strand

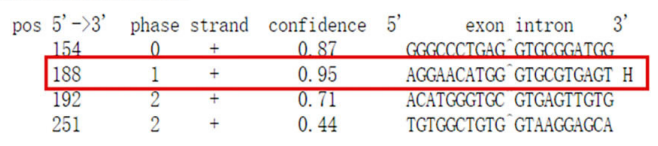

D

\begin{tabular}{|c|c|c|c|c|c|c|c|}
\hline \multirow[b]{2}{*}{ Position (bp) } & \multirow[b]{2}{*}{ Putative splice site } & \multirow[b]{2}{*}{ Sequence } & \multirow[b]{2}{*}{ Score } & \multicolumn{3}{|c|}{ Activations** } & \multirow[b]{2}{*}{ Confidence"* } \\
\hline & & & & Intron GC* & Alt./Cryptic & Constitutive & \\
\hline 90 & Alt. isoform/cryptic donor & CAAGGCTCAGgtggaagagt & 5.957 & 0.614 & 0.784 & 0.163 & 0.792 \\
\hline 122 & Alt. isoform/cryptic donor & ATGGCAGCGAgtaccagtct & 4.718 & 0.614 & 0.881 & 0.089 & 0.899 \\
\hline 141 & Alt isoform/cryptic donor & TGTGAAGCTGgtgggccctg & 10.336 & 0.614 & 0.633 & 0.288 & 0.545 \\
\hline 153 & Alt. isoform/cryptic donor & GGGCCCTGAGgtgcggatgg & 11.186 & 0.586 & 0.572 & 0.341 & 0.403 \\
\hline 187 & Alt isoform/cryptic donor & AGGAACATGGgtgcgtgagt & 8.349 & 0.614 & 0.677 & 0.256 & 0.622 \\
\hline 191 & Alt. isoform/cryptic donor & ACATGGGTGCgtgagttgtg & 7.631 & 0.614 & 0.669 & 0.262 & 0.608 \\
\hline 226 & Alt. isoform/cryptic acceptor & ccactgacagTGGGGGCAGG & 3.989 & 0.586 & 0.894 & 0.099 & 0.889 \\
\hline 250 & Constitutive donor & TGTGGCTGTGgtaaggagca & 10.383 & 0.543 & 0.317 & 0.583 & 0.456 \\
\hline
\end{tabular}

FIGURE 4 | Use prediction software to analyze the variant site. (A) The predict result of wild type by using NetGene2 Server (the red square represented). (B) "AGGAACATGG GTGCGTGAGT" was the appropriate predict result to affects the splicing impact by using NetGene2 Server (the red square represented). (C) The predict result of wild type by using ASSP (the red square represented). (D) The predict result of mutant type by using ASSP (the red square represented).

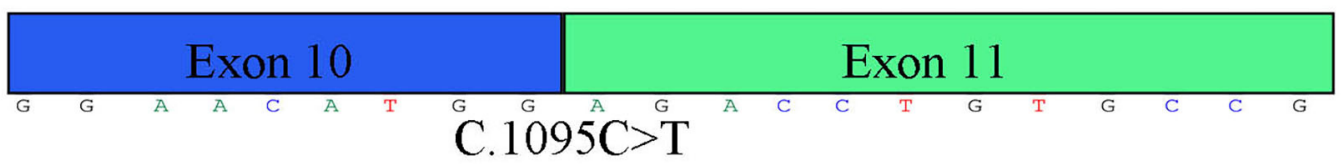

\section{A Proband}

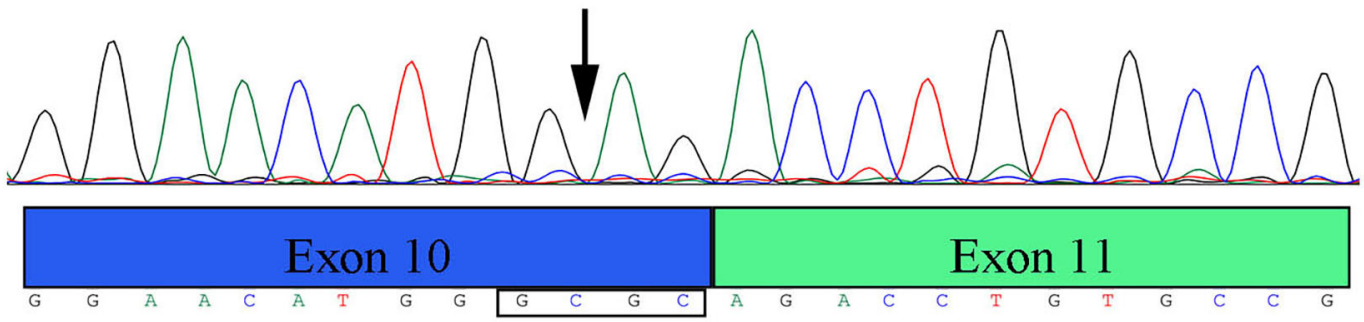

\section{B Father}

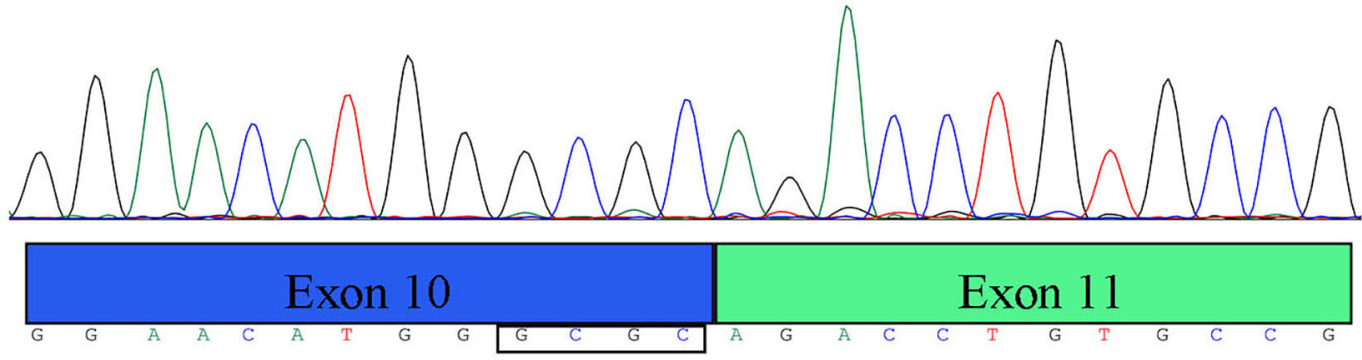

\section{Mother}

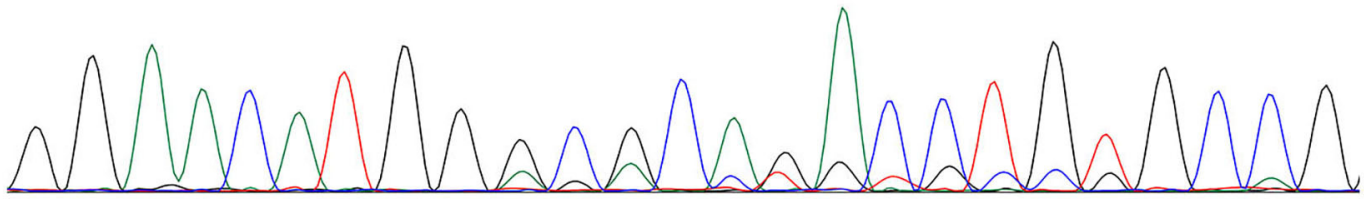

FIGURE 5 | Results of RT-PCR of PLOD1 variant in pedigree. (A) The proband variant resulted in a premature termination codon of exon 10 and affected the expression of the four bases GCGC. (B,C) Parents' CDNA at exon 10 and exon 11.

accompanied by dislocation or subluxation (especially in the shoulders, knees, and hips) (3). There are 10 secondary standards: skin excessive extension; simply bruisable skin; medium-sized artery rupture or aneurysm; osteopenia or osteoporosis; blue sclera; hernia (navel or groin); chest deformity; talipes equinovarus; marfanoid habitus; and refractive errors (myopia or hyperopia). In addition, there are also gene-specific secondary criteria for PLOD1 including skin brittleness (easy bruising, skin fragility, poor wound healing, widening atrophic scars); sclera, and eye brittleness or rupture; microcorneas; 


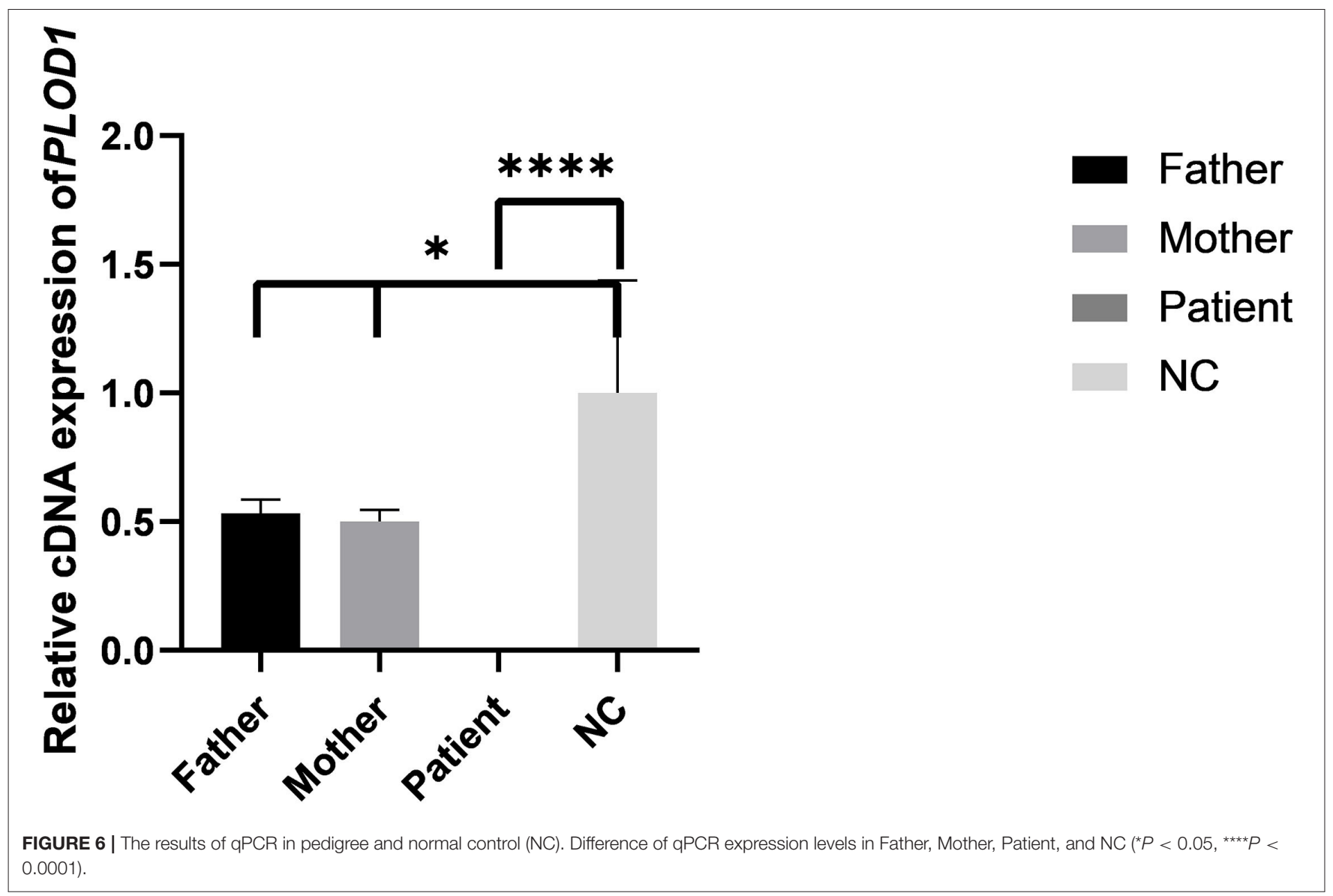

and facial deformities. Patients are required to meet the first two main standards and the third main standard or three secondary standards for a confirmed diagnosis of kEDS-PLOD1 (7). The proband met the two main standards, including congenital muscle weakness; congenital or early-onset kyphosis (progressive or non-progressive); and the three secondary standards including skin excessive extension, simply bruisable skin, and skin brittleness. Especially the genetic detection of PLOD1 variants and kEDS could be diagnosed. As per the latest kEDS-PLOD1 diagnostic criteria of 2017, patients like our proband with kEDS-PLOD1 do not necessarily have kyphosis at birth and infancy (3). According to previous reports, patients will have more complications during their development, such as recurrent pneumonia and cardiac failure (caused by severe kyphoscoliosis), chronic respiratory failure, glaucoma, retinal detachment, arteriorrhexis, arterial aneurysm, and especially circulatory system complications that can be more life-threatening $(2,10)$.

To improve the long-term survival rate of kEDS-PLOD1 and the quality of patients' life, early diagnosis and treatment for patients, along with improving the prognosis and longterm follow-up are crucial. On the basis of previous reports of vascular ruptures, it could be seen more commonly in kEDS-PLOD1. Therefore, we need to take measures to improve the growth and development of blood vessels. For example, regular echocardiographic examinations are used to assess the cardiovascular condition (computed tomography and angiography may be considered if the disease changes further), monitor the size of the aortic root until the patient is 5 years old, and assesses the condition of mitral or tricuspid valve prolapse (11). It is worth noting that the neurological signs and neurodevelopmental intelligence of affected children are generally normal, but learning disabilities have been reported in eight patients (two of whom had reported prenatal or perinatal intracranial hemorrhage) (1, 12). The proband also had intracranial hemorrhage and unexplained ecchymosis on the face, hands, and feet. However, the proband was delivered normally, which does not support the performance of petechial and intracranial hemorrhage caused by birth injury. Further examination of the patient's blood coagulation function level showed normal results, also not supporting the intracranial hemorrhage. In summary, intracranial hemorrhage is related to vascular disease or arterial rupture unique to $\mathrm{kEDS}-P L O D 1$. At present, the follow-up of proband shows that the muscle strength remains poor and the absorption of head hematoma improves. We suggest that in newborns with joint hypermobility, with or without kyphoscoliosis, low muscle strength, skin fragility, and congenital low intraocular pressure, clinicians should highly consider kEDS-PLOD1. 
PLOD1 is located on chromosome 1p36.22 and contains 20 exons. The pathogenic variant in PLOD1 causes kEDS-PLOD1. The PLOD1 gene encodes LH1 and plays an important role in maintaining the stability of intermolecular crosslinking. The LH1 activity of fibroblasts in patients is lower than that in normal people. According to previous reports, $15 \% \mathrm{PLOD}^{-/-}$mice died within 1-4 months because of the degeneration of collagen fibers and abnormal smooth muscle cells in the tissues of living mice, indicating that the mice developed aortic dissection and hence aging and rupture of vascular function (13). In the past reports of the PLOD1 variant, the most common was large duplication of the 10-16 exons that was $>20 \%$ in kEDS-PLOD1 patients, and the others were point variants, insertions, and deletions that caused premature termination of codons and splicing variants caused exon skipping (14). We analyze the characteristics of a genetic and pathogenic variant in PLOD1.

In this research, we verified in detail the genetic information of c.1095C $>\mathrm{T}$ (p. Gly365=) in PLOD1 in a Chinese neonatal pedigree. In this pedigree, the proband had a homozygous variant, and the parents were all heterozygous carriers. The c. $1095 \mathrm{C}>\mathrm{T}$ synonymous variant in PLOD1 introduced a single nucleotide transition from $\mathrm{C}$ to $\mathrm{T}$ at nucleotide 1095 . Based on the ACMG standards and guidelines for the interpretation of sequence variants, c. $1095 \mathrm{C}>\mathrm{T}$ is classified as "VUS" because of insufficient evidence. The most canonical splicing-site variants are located at the $\pm 1,2$ positions of the $5^{\prime}$ or $3^{\prime}$ splice sites (15). Therefore, the pathogenicity of the $\mathrm{c} .1095 \mathrm{C}>\mathrm{T}$ cryptic splice site variant is still unclear. In order to verify the pathogenic evidence that VUS (c.1095C $>$ T) may affect splicing, we conducted a minigene assay on the splicing of PLOD1. Thus, the premature termination codon of exon 10 was confirmed. This conclusion was also confirmed in RT-PCR. As shown by the qPCR results, the synonymous variant affected the splicing of exons, and the mRNA level of the proband was significantly lower than that of the normal control. The mRNA levels of both parents were half of the normal control, but they were phenotypically normal, which might not be high enough to make the parents sick. Hence, the pathogenicity threshold of the disease could be further explored. Interestingly, before in vitro and in vivo experiments, we applied the software to predict the splicing impact and also reached consistent conclusions. An increasing number of research studies have reported that synonymous variants without changing the amino acid sequence could also become dangerous factors for disease (16-18). Variants leading to abnormal splicing of mRNA are understood to be the main pathogenic factors (17). It is estimated that $90 \%$ of disease-related synonymous variants may affect splicing function (19). Recently, the Gene Panel and WES have significantly increased the diagnostic positive rate of rare diseases, enabling genetic diagnosis in $35-50 \%$ cases, and in particular, the application of RNA-seq to the diagnosis of genetic diseases has developed considerably (20).

This variant c. $1095 \mathrm{C}>\mathrm{T}$ (p.Gly365=) has already been named as rs1032781250 (1). In that case report, a 9-year-old male patient had the c.1095C $>\mathrm{T}$ mutation, and the authors hypothesized that the variant caused abnormal splicing of exon 10, which terminated the translation prematurely. However, our study carried out a more comprehensive genetic inspection and verification and described the genetic pathogenic mechanism of splicing on kEDS-PLOD1. The 9-year-old male patient was born with kyphoscoliosis, but he did not get a clear kEDS diagnosis during the neonatal period or the long-term followup, and still could not walk independently. Nevertheless, the proband in our study was born without kyphoscoliosis. We followed up the proband until the time of writing this manuscript and can confirm that the patient has still not developed kyphoscoliosis. We suspect that patients who are not born with kyphoscoliosis will develop this later in life. Therefore, we suggest that when kyphoscoliosis is detected in the neonatal period, relevant auxiliary examinations and genetic testing should be carried out promptly to exclude the differential diagnosis and ultimately improve it to increase the early diagnosis rate of kEDS-PLOD1.

In conclusion, our research verified that the homozygous synonymous variant c.1095 C>T (p.Gly365=) in the PLOD1 gene caused the pathogenic phenotype in the proband. The combined application of WES and functional studies contribute to verifying the effect of uncertain gene variants on splicing, upgrading pathogenicity evidence, and determining the cause of disease. This is helpful for the early diagnosis and treatment of kEDS.

\section{DATA AVAILABILITY STATEMENT}

The datasets for this article are not publicly available due to concerns regarding participant/patient anonymity. Requests to access the datasets should be directed to the corresponding author.

\section{ETHICS STATEMENT}

The studies involving human participants were reviewed and approved by the Ethics and Human Committee of Tianjin Children's Hospital. The patients provided written informed consent to participate in this study.

\section{AUTHOR CONTRIBUTIONS}

$\mathrm{XY}$ and JS conceived the idea, conceptualized the study, and drafted the manuscript. YN and YZ collected the data. PW and WZ analyzed the data. JS and YL reviewed the manuscript. All authors read and approved the final draft.

\section{FUNDING}

This research was funded by the Program of Tianjin Science and Technology Plan (18ZXDBSY00170) and the Project of Tianjin Health Science and Technology (ZC20120 and KJ20166). 


\section{REFERENCES}

1. Rohrbach M, Vandersteen A, Yis U, Serdaroglu G, Ataman E, Chopra M, et al. Phenotypic variability of the kyphoscoliotic type of Ehlers-Danlos syndrome (EDS VIA): clinical, molecular and biochemical delineation. Orphanet J Rare Dis. (2011) 6:46. doi: 10.1186/1750-1172-6-46

2. Beighton P, De Paepe A, Steinmann B, Tsipouras P, Wenstrup RJ. Ehlers-Danlos syndromes: revised nosology, Villefranche, 1997. EhlersDanlos National Foundation (USA) and Ehlers-Danlos Support Group (UK). Am J Med Genet. (1998) 77:31-7. doi: 10.1002/(sici)10968628(19980428)77:1<31::aid-ajmg8>3.0.co;2-o

3. Malfait F, Francomano C, Byers P, et al. The 2017 international classification of the Ehlers-Danlos syndromes. Am J Med Genet C Semin Med Genet. (2017) 175:8-26. doi: 10.1002/ajmg.c.31552

4. Steinmann B, Royce PM, Superti-Furga A. The ehlers-danlos syndrome. In: Royce PM, Steinmann B, editors. Connective Tissue and Its Heritable Disorders. John Wiley and Sons (2000). p. 431-523. doi: 10.1002/0471221929.ch9

5. Hyland J, Ala-Kokko L, Royce P, Steinmann B, Kivirikko KI, Myllyla R. A homozygous stop codon in the lysyl hydroxylase gene in two siblings with Ehlers-Danlos syndrome type VI. Nat Genet. (1992) 2:22831. doi: $10.1038 /$ ng1192-228

6. Steinmann B, Eyre DR, Shao P. Urinary pyridinoline cross-links in Ehlers-Danlos syndrome type VI. Am J Hum Genet. (1995) 57:15058. doi: 10.1002/ajmg.1320590428

7. Conti R, Zanchi C, Barbi E. A floppy infant without lingual frenulum and kyphoscoliosis: Ehlers Danlos syndrome case report. Ital J Pediatr. (2021) 47:28. doi: 10.1186/s13052-021-00984-y

8. Yeowell HN, Walker LC, Farmer B, Heikkinen J, Myllyla R. Mutational analysis of the lysyl hydroxylase 1 gene (PLOD) in six unrelated patients with Ehlers-Danlos syndrome type VI: prenatal exclusion of this disorder in one family. Hum Mutat. (2000) 16:90. doi: 10.1002/10981004(200007)16:1<90::AID-HUMU19>3.0.CO;2-U

9. Richards S, Aziz N, Bale S, Bick D, Das S, Gastier-Foster J, et al. Standards and guidelines for the interpretation of sequence variants: a joint consensus recommendation of the American College of Medical Genetics and Genomics and the Association for Molecular Pathology. Genet Med. (2015) 17:40523. doi: 10.1038/gim.2015.30

10. Jacobs JWG, Cornelissens LJM, Veenhuizen MC. Ehlers-Danlos Syndrome: A Multidisciplinary Approach. Amsterdam: IOS Press (2018). doi: 10.3233/978-1-61499-878-5-i

11. Ghali N, Sobey G, Burrows N. Ehlers-Danlos syndromes. BMJ. (2019) 366:14966. doi: 10.1136/bmj.14966

12. Wenstrup RJ, Murad S, Pinnell SR. Ehlers-Danlos syndrome type VI: clinical manifestations of collagen lysyl hydroxylase deficiency. J Pediatr. (1989) 115:405-9. doi: 10.1016/s0022-3476(89)80839-x
13. Takaluoma K, Hyry M, Lantto J, et al. Tissue-specific changes in the hydroxylysine content and cross-links of collagens and alterations in fibril morphology in lysyl hydroxylase 1 knock-out mice. J Biol Chem. (2007) 282:6588-96. doi: 10.1074/jbc.M608830200

14. Yeowell HN, Walker LC. Mutations in the lysyl hydroxylase 1 gene that result in enzyme deficiency and the clinical phenotype of Ehlers-Danlos syndrome type VI. Mol Genet Metab. (2000) 71:212-24. doi: 10.1006/mgme.2000. 3076

15. Anna, G. Monika, Splicing mutations in human genetic disorders: examples, detection, and confirmation. J. Appl. Genet. (2018) 59:25368. doi: 10.1007/s13353-018-0444-7

16. Hunt RC, Simhadri VL, Iandoli M, Sauna ZE, Kimchi-Sarfaty C. Exposing synonymous mutations. Trends Genet. (2014) 30:308-21. doi: 10.1016/j.tig.2014.04.006

17. Chamary JV, Parmley JL, Hurst LD. Hearing silence: non-neutral evolution at synonymous sites in mammals. Nat Rev Genet. (2006) 7:98-108. doi: 10.1038/nrg1770

18. Sauna ZE, Kimchi-Sarfaty C. Understanding the contribution of synonymous mutations to human disease. Nat Rev Genet. (2011) 12:683-91. doi: 10.1038/nrg3051

19. Mort M, Sterne-Weiler T, Li B, et al. MutPred Splice: machine learningbased prediction of exonic variants that disrupt splicing. Genome Biol. (2014) 15:R19. doi: 10.1186/gb-2014-15-1-r19

20. Fresard L, Smail C, Ferraro NM, et al. Identification of raredisease genes using blood transcriptome sequencing and large control cohorts. Nat Med. (2019) 25:911-9. doi: 10.1038/s41591-0190457-8

Conflict of Interest: The authors declare that the research was conducted in the absence of any commercial or financial relationships that could be construed as a potential conflict of interest.

Publisher's Note: All claims expressed in this article are solely those of the authors and do not necessarily represent those of their affiliated organizations, or those of the publisher, the editors and the reviewers. Any product that may be evaluated in this article, or claim that may be made by its manufacturer, is not guaranteed or endorsed by the publisher.

Copyright (c) 2022 Yan, Shu, Nie, Zhang, Wang, Zhou, Cui and Liu. This is an open-access article distributed under the terms of the Creative Commons Attribution License (CC BY). The use, distribution or reproduction in other forums is permitted, provided the original author(s) and the copyright owner(s) are credited and that the original publication in this journal is cited, in accordance with accepted academic practice. No use, distribution or reproduction is permitted which does not comply with these terms. 